\title{
Bismuth-Based, Disposable Sensor for the Detection of Hydrogen Sulfide Gas
}

Samuel M. Rosolina, Thomas S. Carpenter, Zi-Ling Xue*

Department of Chemistry, University of Tennessee, Knoxville, Tennessee 37996, USA

\section{Supporting Information}

\section{Table of Contents}

Content

Page

- $\quad$ Laboratory Generation of 1.35-L, ppb-ppm $\mathrm{H}_{2} \mathrm{~S}$ Gas and Its Sensing................S-2

- $\quad$ Figure S1. The system to generate $\mathrm{H}_{2} \mathrm{~S}$ gas.............

- $\quad$ Figure S2. Sensors containing differing amounts of Bi(III) after being exposed to $50 \mathrm{ppb}$ gas equivalency NaHS solutions......................... 
Laboratory Generation of 1.35-L, ppb-ppm $\mathrm{H}_{2} \mathrm{~S}$ Gas and Its Sensing. Few reports of devices for generating ppb-ppm $\mathrm{H}_{2} \mathrm{~S}$ gas, especially at a small volume of 1.35-L, have been reported in the literature.

A 1.35-L glass chamber was made in the glass-blowing shop at the University of Tennessee. This volume was chosen to mimic the breath volume of an average human. With a closed top and open bottom, the chamber was designed to sit flat on a stir plate (Figure S1, A). The chamber has one outlet for the generated gas (Figure S1, E), one inlet used for nitrogen gas, followed by water (Figure S1, D), and another inlet attached to a small, internal capillary used for bubbling nitrogen gas (Figure S1, C).

Prior to generation of $\mathrm{H}_{2} \mathrm{~S}$ gas, a small container is filled with $70 \mathrm{~mL}$ of $\mathrm{HCl}$ solution at pH 1. A loosely capped vial containing a NaHS solution and an upright stir bar is then placed, standing, in the container holding the acidic solution. The chamber (Figure S1, A) is placed over top of these containers and sealed tightly to the stir plate using household Vaseline grease. This seal is strong enough to make the entire vessel water tight. To remove all oxygen and avoid the oxidation of $\mathbf{S}^{2-}$ to $\mathbf{S}$, reaction chamber $\mathbf{A}$ is flushed with nitrogen gas through hose $\mathbf{D}$, and the acidic solution is bubbled through the internal capillary attached to hose $\mathbf{C}$ for $10 \mathrm{~min}$. The ultrapure DI water in container $\mathbf{B}$ is simultaneously bubbled with nitrogen gas as well.

A sensor, previously dampened with $80 \mu \mathrm{L}$ of $0.1 \mathrm{M} \mathrm{NaOH}$, is placed on a wire mesh as the sensor stand (Figure S1, F). A plastic micro pipette tip has been firmly attached and sealed to hose $\mathbf{E}$ and funnels the generated gas to a smaller area (roughly a $3 \mathrm{~mm}$ diameter) directly onto the surface of the sensor. This pipette tip is held so that it is in direct contact with the sensor. The nitrogen gas is clamped off at hose $\mathbf{D}$ and lowered to a very slow flow through hose C. The stir plate is then turned on at a high rate, knocking the vial of the NaHS solution into the 
$\mathrm{HCl}$ solution and forming $\mathrm{H}_{2} \mathrm{~S}$ gas (Eq. 4). At this point the water (also bubbled with $\mathrm{N}_{2}$ ) of container $\mathbf{B}$ is allowed to slowly travel into the reaction chamber $\mathbf{A}$ via hose $\mathbf{D}$ using gravitational flow. As chamber $\mathbf{A}$ is filled with water, it pushes out the generated $\mathrm{H}_{2} \mathrm{~S}$ gas through hose $\mathbf{E}$ onto the sensor at $\mathbf{F}$. Direct contact between the pipette tip of hose $\mathbf{E}$ and the sensor at $\mathbf{F}$ forces the gas through the sensor, allowing for more interaction between $\mathrm{Bi}^{3+}$ and $\mathrm{S}^{2-}$. The acidity of solution in the container is enough that even when diluted to $1.35 \mathrm{~L}$ after mixing, its $\mathrm{pH}$ is still 3 .

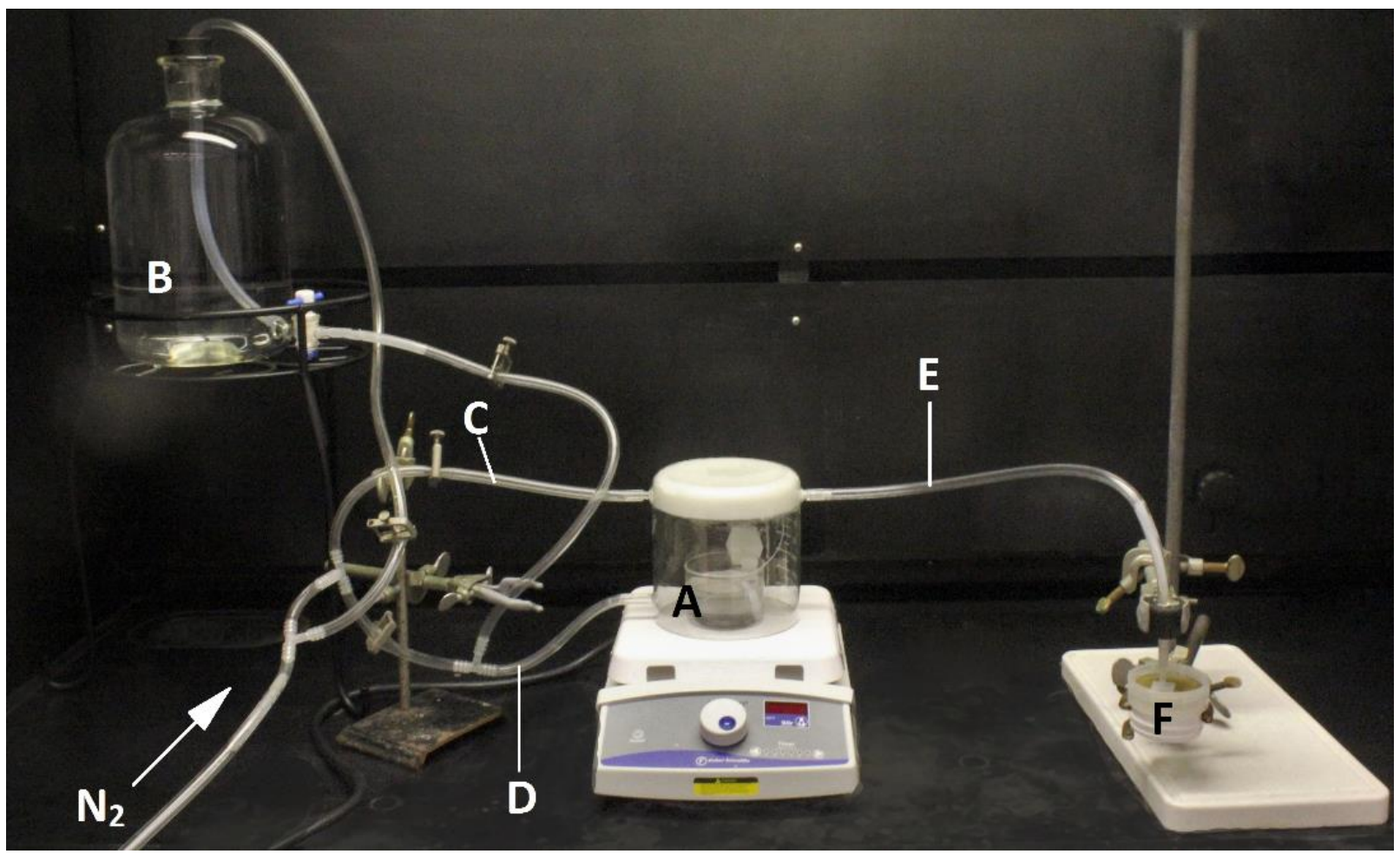

Figure S1. The system to generate $\mathrm{H}_{2} \mathrm{~S}$ gas. A: Reaction chamber; B: Water container; $\mathbf{C}: \mathrm{N}_{2^{-}}$ inlet hose; D: Water-inlet hose; E: Gas-outlet hose; F: Wire mesh as the sensor stand. 


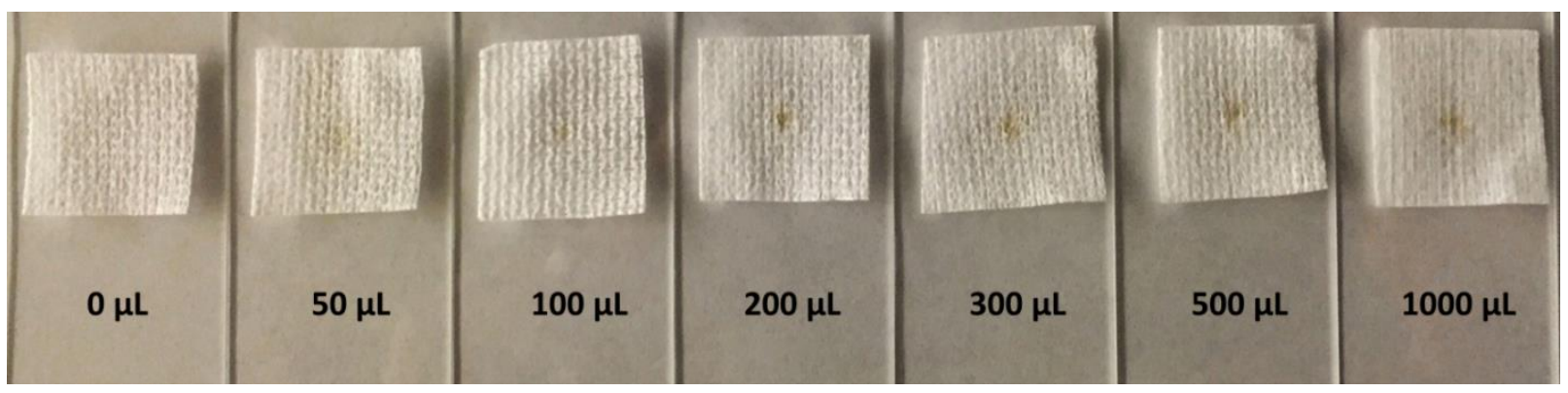

Figure S2. Sensors containing differing amounts of Bi(III) after being exposed to $50 \mathrm{ppb}$ gas equivalency NaHS solutions. Volumes of $0,50,100,200,300,500$, and $1000 \mu \mathrm{L}$ of $\mathrm{Bi}\left(\mathrm{NO}_{3}\right)_{3}$ /acetone suspensions were deposited on the Prozorb squares. Note no spot development for the Prozorb containing no Bi. 